\title{
A New Approach to Asymptotic Behavior for a Finite Element Approximation in Parabolic Variational Inequalities
}

\author{
Salah Boulaaras ${ }^{1,2}$ and Mohamed Haiour ${ }^{1,2}$ \\ ${ }^{1}$ Hydrometeorological Institute of Formation and Research, Box. 7019, Seddikia, Oran 31000, Algeria \\ ${ }^{2}$ Department of Mathematics, Faculty of Science, University of Annaba, Box. 12, Annaba 23000, Algeria \\ Correspondence should be addressed to Salah Boulaaras, saleh_boulaares@yahoo.fr
}

Received 30 March 2011; Accepted 13 May 2011

Academic Editors: G. Garcea and S. Zhang

Copyright (c) 2011 S. Boulaaras and M. Haiour. This is an open access article distributed under the Creative Commons Attribution License, which permits unrestricted use, distribution, and reproduction in any medium, provided the original work is properly cited.

The paper deals with the theta time scheme combined with a finite element spatial approximation of parabolic variational inequalities. The parabolic variational inequalities are transformed into noncoercive elliptic variational inequalities. A simple result to time energy behavior is proved, and a new iterative discrete algorithm is proposed to show the existence and uniqueness. Moreover, its convergence is established. Furthermore, a simple proof to asymptotic behavior in uniform norm is given.

\section{Introduction}

A great work has been done on questions of existence and uniqueness for parabolic variational and quasivariational inequalities over the last three decades. However, very much remains to be done on the numerical analysis side, especially error estimates and asymptotic behavior for the free boundary problems (cf., e.g., [1-8]).

In this paper, we propose a new iterative discrete algorithm to prove the existence and uniqueness, and we devote the asymptotic behavior using the $\theta$ time scheme combined with a finite element spatial approximation for parabolic inequalities.

Let us assume that $K$ is an implicit convex set defined as follows:

$$
K=\left\{v(t, x) \in L^{2}\left(0, T, H_{0}^{1}(\Omega)\right), v(t, x) \leq \psi(t, x), v(0, x)=v_{0} \text { in } \Omega\right\},
$$

with

$$
\psi \in L^{2}\left(0, T, W^{2, \infty}(\Omega)\right)
$$


We consider the following problem, find $u \in K$ solution of

$$
\begin{gathered}
\frac{\partial u}{\partial t}+A u \leq f \quad \text { in } \Sigma \\
u(t, x)=0 \quad \text { in } \Gamma
\end{gathered}
$$

where $\Sigma$ is a set in $\mathbb{R} \times \mathbb{R}^{N}$ defined as $\Sigma=\Omega \times[0, T]$ with $T<+\infty$, and $\Omega$ is convex domain in $\mathbb{R}^{N}$, with sufficiently smooth boundary $\Gamma$.

The symbol $(\cdot, \cdot)$ stands for the inner product in $L^{2}(\Omega)$, and $A$ is an operator defined over $H^{1}(\Omega)$ by

$$
A u=-\sum_{i j=1}^{N} \frac{\partial}{\partial x_{i}} a_{i j}(x) \frac{\partial u}{\partial x_{j}}+\sum_{j=1}^{N} b_{j}(x) \frac{\partial u}{\partial x_{j}}+a_{0}(x) u
$$

and whose coefficients: $a_{i, j}(x), b_{j}(x), a_{0}(x) \in L^{\infty}(\Omega) \cap C_{2}(\bar{\Omega}), x \in \bar{\Omega}, 1 \leq i, j \leq N$ are sufficiently smooth functions and satisfy the following conditions:

$$
\begin{gathered}
a_{i j}(x)=a_{j i}(x) ; \quad a_{0}(x) \geq \beta>0, \quad \beta \text { is a constant. } \\
\sum_{i j=1}^{N} a_{i j}(x) \xi_{i} \xi_{j} \geq \gamma|\xi|^{2} ; \quad \xi \in \mathbb{R}^{N}, \gamma>0, \quad x \in \bar{\Omega} .
\end{gathered}
$$

$f$ is a regular functions satisfying

$$
f \in L^{2}\left(0, T, L^{\infty}(\Omega)\right) \cap C^{1}\left(0, T, H^{-1}(\Omega)\right), \quad f \geq 0
$$

We specify the following notations:

$$
\|\cdot\|_{L^{2}(\Omega)}=\|\cdot\|_{2}, \quad\|\cdot\|_{1}=\|\cdot\|_{H_{0}^{1}(\Omega)}, \quad\|\cdot\|_{L^{\infty}(\Omega)}=\|\cdot\|_{\infty} .
$$

As we have said before, the aim of the present paper is to show that the asymptotic behavior can be properly approximated by a $\theta$ time scheme combined with a finite element spatial using a new iterative algorithm. We precede our analysis in two steps: in the first step, we discretize in space; that is, we approach the space $H_{0}^{1}$ by a space discretization of finite dimensional $V^{h} \subset H_{0}^{1}$. In the second step, we discretize the problem with respect to time using the $\theta$-scheme. Therefore, we search a sequence of elements $u_{h}^{n} \in V^{h}$ which approaches $u^{n}\left(t_{n}\right), t_{n}=n \Delta t$, with initial data $u_{h}^{0}=u_{0 h}$. Our approach stands on a discrete stability result and error estimate for parabolic variational inequalities.

The paper is organized as follows. In Section 2, we prove the simple result to time energy behavior of the semidiscrete parabolic variational inequalities. In Section 3, we prove the $L^{\infty}$-stability analysis of the $\theta$-scheme for P.V.I, and finally, in Section 4 , we first associate with the discrete P.V.I problem a fixed point mapping, and we use that in proving the existence of a unique discrete solution, and later, we establish the asymptotic behavior estimate of $\theta$-scheme by the uniform norm for the problem studied. 


\section{Priory Estimate of the Discrete Parabolic Variational Inequalities}

We can reformulate (1.3) to the following variational inequality:

$$
\left(\frac{\partial u}{\partial t}, v-u\right)+a(u, v-u) \geq(f, v-u), \quad v \in K,
$$

where $a(\cdot, \cdot)$ is the bilinear form associated with operator $A$ defined in (1.4). Namely,

$$
a(u, v)=\int_{\Omega}\left(\sum_{i j=1}^{N} a_{i j}(x) \frac{\partial u}{\partial x_{i}} \frac{\partial v}{\partial x_{j}}+\sum_{j=1}^{N} b_{j}(x) \frac{\partial u}{\partial x_{j}} v+a_{0}(x) u v\right) d x
$$

Theorem 2.1 (see [9]). The problem (1.3) has an unique solution $u \in K(u)$. Moreover, one has

$$
u \in L^{2}\left(0, T ; H_{0}^{1}(\Omega)\right), \quad \frac{\partial u}{\partial t} \in L^{2}\left(0, T ; H^{-1}(\Omega)\right) .
$$

Lemma 2.2 (Sobolev-Poincare inequality). Let $\Omega$ be a bounded overt in $\mathbb{R}^{N}$, with sufficiently smooth boundary $\Gamma$, then there exists a $C_{*}$ such that

$$
\|u\|_{2} \leq C_{*}\|\nabla u\|_{2}, \quad v \in H_{0}^{1}(\Omega) \cap C^{2}(\bar{\Omega}), \quad \nabla=\sum_{i=1}^{N} \frac{\partial}{\partial x_{i}} .
$$

\subsection{The Discrete Problem}

Let us assume that $\Omega$ can be decomposed into triangles and $\tau_{h}$ denotes the set of all the elements $h>0$, where $h$ is the mesh size. We assume that the family $\tau_{h}$ is regular and quasiuniform, and we consider the usual basis of affine functions $\varphi_{i}, i=\{1, \ldots, m(h)\}$ defined by $\varphi_{i}\left(M_{j}\right)=\delta_{i j}$, where $M_{j}$ is a vertex of the considered triangulation. We introduce the following discrete spaces $V^{h}$ of finite element:

$$
\begin{gathered}
V_{h}=\left\{v_{h} \in L^{2}\left(0, T, H_{0}^{1}(\Omega)\right) \cap C\left(0, T, H_{0}^{1}(\bar{\Omega})\right), \text { such that }\left.v_{h}\right|_{k} \in P_{1}, k \in \tau_{h},\right. \\
\left.v_{h} \leq r_{h} \psi, v_{h}(\cdot, 0)=v_{h 0} \text { in } \Omega .\right\} .
\end{gathered}
$$

We consider $r_{h}$ to be the usual interpolation operator defined by

$$
v \in L^{2}\left(0, T, H_{0}^{1}(\Omega)\right) \cap C\left(0, T, H_{0}^{1}(\bar{\Omega})\right), \quad r_{h} v=\sum_{i=1}^{m(h)} v\left(M_{i}\right) \varphi_{i}(x) .
$$

The Discrete Maximum Principle Assumption (see [10])

The matrix whose coefficients $a\left(\varphi_{i}, \varphi_{j}\right)$ are supposed to be $M$-matrix. For convenience, in all the sequels, $C$ will be a generic constant independent on $h$. 


\subsubsection{Priory Estimate}

Theorem 2.3. Let us assume that the discrete bilinear form $a(\cdot, \cdot)$ defined as (2.2) is weakly coercive in $V^{h} \subset H_{0}^{1}(\Omega)$. Then, there exists two constants $\alpha>0$ and $\lambda>0$ such that

$$
a\left(u_{h}, u_{h}\right)+\lambda\left\|u_{h}\right\|_{2} \geq \alpha\left\|u_{h}\right\|_{1}
$$

where

$$
\lambda=\left(\frac{\left\|b_{j}\right\|_{\infty}^{2}}{2 \gamma}+\frac{\gamma}{2}+\left\|a_{0}\right\|_{\infty}\right), \quad \alpha=\frac{\gamma}{2}
$$

Proof. The bilinear form $a(\cdot, \cdot)$ is defined by

$$
a\left(u_{h}, u_{h}\right)=\int_{\Omega}\left(\sum_{i j=1}^{N} a_{i j}(x) \frac{\partial u_{h}}{\partial x_{i}} \frac{\partial u_{h}}{\partial x_{j}}+\sum_{j=1}^{N} b_{j}(x) \frac{\partial u_{h}}{\partial x_{j}} u_{h}+a_{0}(x) u_{h}^{2}\right) d x
$$

under assumption (1.6), we have

$$
\sum_{i j=1}^{N} \int_{\Omega} a_{i j}(x) \frac{\partial u_{h}}{\partial x_{i}} \frac{\partial u_{h}}{\partial x_{j}}>r \sum_{i=1}^{N}\left(\int_{\Omega} \frac{\partial u_{h}}{\partial x_{i}}\right)^{2}=r\left\|\nabla u_{h}\right\|_{2}^{2}
$$

and since

$$
\begin{aligned}
\left|\sum_{j=1}^{N} \int_{\Omega} b_{j}(x) \frac{\partial u_{h}}{\partial x_{j}} u_{h}\right| & \leq \sup _{j}\left|b_{j}\right| \sum_{j=1}^{N} \int_{\Omega}\left|\frac{\partial u_{h}}{\partial x_{j}} u_{h}\right| \\
& \leq\left\|b_{j}\right\|_{\infty}\left\|\nabla u_{h}\right\|_{2}\left\|u_{h}\right\|_{2},
\end{aligned}
$$

then we make use of the algebraic inequality

$$
a b \leq \frac{1}{2}\left(a^{2}+b^{2}\right), \quad \forall a, b \in \mathbb{R}, \forall \gamma>0,
$$

and choosing

$$
\begin{aligned}
& a=\left\|\nabla u_{h}\right\|_{2} \cdot \sqrt{\gamma}, \\
& b=\frac{\left\|b_{j}\right\|_{\infty}}{\sqrt{\gamma}}\left\|u_{h}\right\|_{2},
\end{aligned}
$$


then we end up with

$$
\left|\sum_{j=1}^{N} \int_{\Omega} b_{j}(x) \frac{\partial u_{h}}{\partial x_{j}} u_{h}\right| \geq-\left(\frac{\gamma}{2}\left\|\nabla u_{h}\right\|_{2}^{2}+\frac{\left\|b_{j}\right\|_{\infty} \cdot\left\|u_{h}\right\|_{2}}{2 \gamma}\right)
$$

so we get

$$
a\left(u_{h}, u_{h}\right) \geq \gamma\left\|\nabla u_{h}\right\|_{2}^{2}-\frac{\gamma}{2}\left\|\nabla u_{h}\right\|_{2}^{2}+\frac{\left\|b_{j}\right\|_{\infty}}{2 \gamma}\left\|u_{h}\right\|_{2}-\left\|a_{0}\right\|_{\infty}\left\|u_{h}\right\|_{2}^{2} .
$$

It can easily verified that

$$
a\left(u_{h}, u_{h}\right) \geq \frac{\gamma}{2}\left(\left\|\nabla u_{h}\right\|_{2}+\left\|u_{h}\right\|_{2}^{2}\right)-\left(\frac{\left\|a_{j}\right\|_{\infty}^{2}}{2 \gamma}+\frac{\gamma}{2}+\left\|a_{0}\right\|_{\infty}\right)\left\|u_{h}\right\|_{2}^{2}
$$

Consequently, we deduce from above that

$$
a\left(u_{h}, u_{h}\right)+\lambda\left\|u_{h}\right\|_{2}^{2} \geq \alpha\left\|u_{h}\right\|_{1}^{2} \quad \text { such that } \alpha=\frac{\gamma}{2}, \lambda=\left(\frac{\left\|a_{j}\right\|_{\infty}^{2}}{2 \gamma}+\frac{\gamma}{2}+\left\|a_{0}\right\|_{\infty}\right) .
$$

We can identify the following result on the time energy behavior:

$$
E_{h}(t)=\int_{\Omega} u_{h}^{2} d x
$$

Setting $v=0$ on (2.1) and after discretization by the finite element in the $V^{h}$, we have the semidiscretization problem

$$
\left(\frac{\partial u_{h}}{\partial t}, u_{h}\right)+a\left(u_{h}, u_{h}\right)=\frac{1}{2} \int_{\Omega} \frac{\partial u_{h}^{2}}{\partial t} d x+a\left(u_{h}, u_{h}\right) \leq\left(f, u_{h}\right) .
$$

Using Theorem 2.3, we deduce that

$$
\begin{aligned}
\frac{1}{2} \int_{\Omega} \frac{d u_{h}^{2}}{d t} d x+a\left(u_{h}, u_{h}\right) & \geq \frac{1}{2} \frac{d}{d t} \int_{\Omega} u_{h}^{2} d x+\alpha\left\|u_{h}\right\|_{1}^{2}-\lambda\left\|u_{h}\right\|_{2}^{2} \\
& =\frac{1}{2} \frac{d}{d t} \int_{\Omega} u_{h}^{2} d x+\alpha\left\|u_{h}\right\|_{2}^{2}+\alpha\left\|\nabla u_{h}\right\|_{2}^{2}-\lambda\left\|u_{h}\right\|_{2}^{2} \\
& =\frac{1}{2}\left(\frac{d}{d t} E_{h}(t)+2(\alpha-\lambda) E_{h}(t)+2 \alpha\left\|\nabla u_{h}\right\|_{2}^{2} d x\right) \\
& \geq \frac{1}{2}\left(\frac{d}{d t} E_{h}(t)+2(\alpha-\lambda) E_{h}(t)+\frac{2 \alpha}{C_{*}^{2}} \int_{\Omega} u_{h}^{2} d x\right) .
\end{aligned}
$$


Thus, we have

$$
\frac{d}{d t} \int_{\Omega} u_{h}^{2} d x+\alpha\left\|u_{h}\right\|_{1}^{2}-\lambda\left\|u_{h}\right\|_{2}^{2} \geq \frac{d}{d t}\left(E_{h}(t)\right)+2\left(\alpha-\lambda+\frac{\alpha}{C_{*}^{2}}\right) E_{h}(t)
$$

Applying the Cauchy-Schwartz inequality on the right-hand side of (2.1), we find

$$
\left(f, u_{h}\right)=\int_{\Omega} f(x, t) u_{h}(x, t) d x \leq\|f\|_{2}\left\|u_{h}\right\|_{2}
$$

So that

$$
\frac{d}{d t} E_{h}(t)+2\left(\alpha-\lambda+\frac{\alpha}{C_{*}^{2}}\right) E_{h}(t) \leq 2\|f\|_{2}\left\|u_{h}\right\|_{2}
$$

Using Young's inequality

$$
a b \leq \varepsilon a^{2}+\frac{1}{4 \varepsilon} b^{2}, \forall a, b \in \mathbb{R}, \forall \varepsilon>0
$$

Thus, we obtain

$$
\frac{d}{d t} E_{h}(t)+2\left(\alpha-\lambda+\frac{\alpha}{C_{*}^{2}}\right) E_{h}(t) \leq 2 \varepsilon E_{h}(t)+\frac{1}{2 \varepsilon}\|f\|_{2}^{2}
$$

taking $\eta=\alpha-\lambda+\alpha / C_{*}^{2}$, thus we have

$$
\frac{d}{d t} E_{h}(t)+2(\eta-\varepsilon) E_{h}(t) \leq \frac{1}{2 \varepsilon}\|f\|_{2}^{2}
$$

Or, equivalently

$$
\left(e^{2(\eta-\varepsilon) t} E_{h}(t)\right)^{\prime} \leq \frac{1}{2 \varepsilon} e^{2(\eta-\varepsilon) t} \int_{\Omega}(f(x, t))^{2} d x
$$

Integrating the last inequality from 0 to $t$, we get

$$
E_{h}(t) \leq e^{-2(\eta-\varepsilon) t} E_{h}(0)+\frac{1}{2 \varepsilon} \int_{0}^{t}\left[e^{2(\eta-\varepsilon)(s-t)}\left(\int_{\Omega}(f(x, s))^{2} d x\right)\right] d s
$$

Remark 2.4. In particular, when $f=0$ and choosing $\varepsilon<\eta$, then (2.28) shows that the energy $E(t)$ decreasing exponentially fast in time. 


\section{The $\theta$-Scheme Method for the Parabolic Variational Inequalities}

\subsection{Stability Analysis for the P.V.I}

We apply the finite element method to approximate inequality (2.1), and the discrete P.V.I takes the form of

$$
\left(\frac{\partial u_{h}}{\partial t}, v_{h}-u_{h}\right)+a\left(u_{h}, v_{h}-u_{h}\right) \geq\left(f, v_{h}-u_{h}\right), \quad v_{h} \in V^{h}
$$

Now, we apply the $\theta$-scheme on the semidiscrete problem (3.1); for any $\theta \in[0,1]$ and $k=1, \ldots, n$, we have

$$
\left(u_{h}^{k}-u_{h}^{k-1}, v_{h}-u_{h}^{\theta, k}\right)+\Delta t \cdot a\left(u_{h}^{\theta, k}, v_{h}-u_{h}^{\theta, k}\right) \geq \Delta t \cdot\left(f^{\theta, k}, v_{h}-u_{h}^{\theta, k}\right), \quad v_{h} \in V^{h},
$$

where

$$
\begin{gathered}
u_{h}^{\theta, k}=\theta u_{h}^{k}+(1-\theta) u_{h}^{k-1}, \\
f^{\theta, k}=\theta f\left(t_{k}\right)+(1-\theta) f\left(t_{k-1}\right) .
\end{gathered}
$$

It is possible to analyze the stability by taking the advantage of the structure of eigenvalues of the bilinear form $a(\cdot, \cdot)$. We recall that $W$ is compactly embedded in $L^{2}(\Omega)$, since $\Omega$ is bounded. Thus, there exists a nondecreasing sequence of eigenvalues $\delta \leq \lambda_{1} \leq \lambda_{2} \leq$ $\cdots$ for the bilinear form $a(\cdot, \cdot)$ satisfying

$$
\omega_{j} \in L^{2}, \quad \omega_{j} \neq 0: a\left(\omega_{j}, v_{h}\right)=\lambda_{j}\left(\omega_{j}, v_{h}\right), \quad \forall v_{h} \in V^{h} .
$$

The corresponding eigenfunctions $\left\{\omega_{j}\right\}$ form a complete orthonormal basis in $L^{2}(\Omega)$. In analogous way, when considering the finite dimensional problem in $W^{h}$, we find a sequence of eigenvalues $\delta \leq \lambda_{1 h} \leq \lambda_{2 h} \leq \cdots \leq \lambda_{m(h)}$ and $L^{2}$-orthonormal basis of eigenvectorss $\omega_{i h} \in W^{h}, i=1,2, \ldots, m(h)$. Any function $v_{h}$ in $V^{h}$ can thus be expanded with respect to the system $\omega_{i h}$ as

$$
v_{h}=\sum_{i=1}^{m(h)}\left(v_{h}, \omega_{i h}\right) \omega_{i h}
$$

in particular, we have

$$
u_{h}^{k}=\sum_{i=1}^{m(h)} u_{i}^{k} \omega_{i h}, \quad u_{i}^{k}=\left(u_{h^{\prime}}^{k} \omega_{i h}\right)
$$

Moreover, let $f_{h}^{k}$ be the $L^{2}$-orthogonal projection of $f^{\theta, k}$ into $W^{h}$, that is, $f_{h}^{k} \in W^{h}$ and

$$
\left(f_{h}^{k}, v_{h}\right)=\left(f^{\theta, k}, v_{h}\right)
$$


and set

$$
f_{h}^{k}=\sum_{i=1}^{m(h)} f_{i}^{k} \omega_{i h}, \quad f_{i}^{k}=\left(f_{h}^{k}, \omega_{i h}\right) .
$$

We are now in a position to prove the stability for $\theta \in[0,1 / 2[$

Choosing in (3.1) $v_{h}=0$, thus we have

$$
\frac{1}{\Delta t}\left(u_{h}^{k}-u_{h}^{k-1}, u_{h}^{\theta, k}\right)+a\left(u_{h}^{\theta, k}, u_{h}^{\theta, k}\right) \leq\left(f^{\theta, k}, u_{h}^{\theta, k}\right), \quad u_{h}^{\theta, k} \in V^{h} .
$$

The inequalities (3.2) is equivalent to

$$
\frac{1}{\Delta t}\left(u_{i}^{k}-u_{i}^{k-1}\right)+\lambda_{i h}\left(\theta u_{i}^{k}+(1-\theta) u_{i}^{k-1}\right) \leq f_{i}^{k}
$$

Since $\omega_{i h}$ are the eigenfunctions means

$$
a\left(\omega_{i h}, \omega_{i h}\right)=\lambda_{i h}\left(\omega_{i h}, \omega_{i h}\right)=\lambda_{i h} \cdot \delta_{i i}=\lambda_{i h},
$$

for each $k=0, \ldots, m(h)-1$, we can rewrite (3.9) as

$$
u_{i}^{k} \leq \frac{1-(1-\theta) \cdot \Delta t \cdot \lambda_{i h}}{1+\theta \Delta t \cdot \lambda_{i h}} u_{i}^{k-1}+\frac{\Delta t}{1+\theta \Delta t \cdot \lambda_{i h}} f_{i}^{k},
$$

this inequality system stable if and only if

$$
\left|\frac{1-(1-\theta) \cdot \Delta t \cdot \lambda_{i h}}{1+\theta \Delta t \cdot \lambda_{i h}}\right|<1
$$

that is to say

$$
2 \theta-1>-\frac{2}{\lambda_{i h} \cdot \Delta t}
$$

means

$$
\Delta t<\frac{2}{(1-2 \theta) \lambda_{i h}}
$$

So that this relation satisfied for all the eigenvalues $\lambda_{i h}$ of bilinear form $a(\cdot, \cdot)$, we have to choose their highest value, and we take it for $\lambda_{m h}=\rho(A)$ (rayon spectral)

We deduce that if $\theta \geq 1 / 2$ the $\theta$-scheme way is stable unconditionally (i.e., stable for all $\Delta t$ ). However, if $0 \leq \theta<1 / 2$ the $\theta$-scheme is stable unless

$$
\Delta t<\frac{2}{(1-2 \theta) \rho(A)} .
$$


We can prove that there exist two positive constants $c_{1}, c_{2}$ such that

$$
\frac{c_{1}}{h^{2}} \leq \lambda_{m h}=c_{2} h^{-2}
$$

thus the method of $\theta$-scheme is stable if and only if

$$
\Delta t<\frac{2 C}{(1-2 \theta)} h^{2}
$$

Notice that this condition is always satisfied if $0 \leq \theta<1 / 2$. Hence, taking the absolute value of (3.12), we have

$$
\left|u_{i}^{m}\right|<\left|u_{i}^{0}\right|+\frac{\Delta t}{1+\theta \Delta t \cdot \lambda_{i h}} \sum_{i=1}^{m-1} f_{i}^{k}
$$

also we deduce that

$$
\left\|u_{i}^{m}\right\|_{\infty}<\left\|u_{i}^{0}\right\|_{\infty}+\left\|\frac{\Delta t}{1+\theta \Delta t \cdot \lambda_{i h}}\right\|_{\infty} \sum_{i=1}^{m-1}\left\|f_{i}^{k}\right\|_{\infty} .
$$

Remark 3.1 (cf. [4]). We assume that the coerciveness assumption (Theorem 2.3) is satisfied with $\lambda=0$, and for each $k=1, \ldots, n$, we find

$$
\left\|u_{h}^{k}\right\|_{2}^{2}+2 \Delta t \sum_{k=1}^{n} a\left(u_{h}^{\theta, k}, u_{h}^{\theta, k}\right) \leq C(n)\left(\sum_{k=1}^{n} \Delta t\left\|f^{\theta, k}\right\|_{2}^{2}\right) .
$$

\section{Asymptotic Behavior of $\theta$-Scheme for the P.V.I}

This section is devoted to the proof of the main result of the present paper; we need first to study some properties such as proving the existence and uniqueness for parabolic variational inequalities.

\subsection{Existence and Uniqueness for P.V.I}

Theorem 4.1 (cf. $[2,3]$ ). Under the previous assumptions, and the maximum principle, there exists a constant $C$ independent of $h$ such that

$$
\left\|u^{\infty}-u_{h}^{\infty}\right\|_{\infty} \leq C h^{2}|\log h|^{2}
$$


where $u^{\infty}$ and $u_{h}^{\infty}$ are, respectively, stationery solutions to the following continue and discrete inequalities:

$$
\begin{aligned}
& b\left(u^{\infty}, v-u^{\infty}\right) \geq\left(f+\lambda u^{\infty}, v-u^{\infty}\right), \quad v \in H_{0}^{1}(\Omega), \\
& b\left(u_{h}^{\infty}, v_{h}-u_{h}^{\infty}\right) \geq\left(f+\lambda u^{\infty}, v_{h}-u_{h}^{\infty}\right), \quad v_{h} \in V^{h},
\end{aligned}
$$

such that

$$
b(\cdot, \cdot)=a(\cdot, \cdot)+\lambda(\cdot, \cdot),
$$

where $\lambda$ is a positive constant arbitrary. We have $u_{h}^{\theta, k}=\theta u_{h}^{k}+(1-\theta) u_{h}^{k-1} \leq \theta r_{h} \psi+(1-\theta) r_{h} \psi=$ $r_{h} \psi$.Thus, we can rewrite (3.1) as, for $u_{h}^{\theta, k} \in V^{h}$

$$
\left(\frac{u_{h}^{\theta, k}}{\theta \Delta t}, v_{h}-\tilde{u}_{h}^{k}\right)+a\left(u_{h}^{\theta, k}, v_{h}-u_{h}^{\theta, k}\right) \geq\left(f^{\theta, k}+\frac{u_{h}^{k-1}}{\theta \Delta t}, v_{h}-u_{h}^{\theta, k}\right), \quad v_{h} \in V^{h}
$$

Thus, our problem (4.5) is equivalent to the following noncoercive elliptic variational inequalities:

$$
b\left(u_{h}^{\theta, k}, v_{h}-\tilde{u}_{h}^{k}\right) \geq\left(f^{\theta, k}+\mu u_{h}^{k-1}, v_{h}-u_{h}^{\theta, k}\right), \quad v_{h} \in V^{h},
$$

such that

$$
\begin{gathered}
b\left(u_{h}^{\theta, k}, v_{h}-u_{h}^{\theta, k}\right)=\mu\left(u_{h}^{\theta, k}, v_{h}-u_{h}^{\theta, k}\right)+a\left(u_{h}^{\theta, k}, v_{h}-u_{h}^{\theta, k}\right), \quad v_{h}, u_{h}^{\theta, k} \in V^{h}, \\
\mu=\frac{1}{\theta \Delta t}=\frac{T}{\theta k^{\prime}}
\end{gathered}
$$

where $u_{h}^{\theta, 1}$ is the solution to the following discrete inequality:

$$
a\left(u_{h}^{\theta, 1}, v_{h}-u_{h}^{\theta, 1}\right)=\left(g\left(t_{k}\right), v_{h}-u_{h}^{\theta, 1}\right), \quad v_{h} \in V^{h},
$$

where $g\left(t_{k}\right)$ is a regular function given.

\subsubsection{A Fixed Point Mapping Associated with Discrete Problem (4.7)}

We consider the mapping

$$
\begin{gathered}
T_{h}: L_{+}^{\infty}(\Omega) \longrightarrow V^{h} \\
w \longrightarrow T_{h}(w)=\xi_{h},
\end{gathered}
$$


where $\xi_{h}$ is the unique solution of the following P.V.I: find $\xi_{h} \in V^{h}$

$$
b\left(\xi_{h}, v_{h}-\xi_{h}\right) \geq\left(f^{\theta, k}+\mu w, v_{h}-\xi_{h}\right), \quad v_{h} \in V^{h} .
$$

Proposition 4.2. Under the previous hypotheses and notations, if one sets $\theta \geq 1 / 2$, the mapping $T_{h}$ is a contraction in $L^{\infty}(\Omega)$ with rate of contraction $1 /(1+\beta \cdot \theta \cdot \Delta t)$. Therefore, $T_{h}$ admits a unique fixed point which coincides with the solution of P.V.I (4.7).

Proof. For $w, \widetilde{w}$ in $L^{\infty}(\Omega)$, we consider $\xi_{h}=T_{h}(w)=\partial\left(f^{\theta, k}+\mu w, r_{h} \psi\right)$ and $\widetilde{\xi}_{h}=T_{h}(\widetilde{w})=$ $\partial\left(f^{\theta, k}+\mu \tilde{w}, r_{h} \widetilde{\psi}\right)$ solution to quasivariational inequalities (4.7) with right-hand side $F^{\theta, k}=$ $f^{\theta, k}+\mu w_{h}, \tilde{F}^{\theta, k}=f^{\theta, k}+\mu \tilde{w}_{h}$.

Now, setting

$$
\phi=\frac{1}{\beta+\mu}\left\|F^{\theta, k}-\tilde{F}^{\theta, k}\right\|_{\infty}
$$

then for $\xi_{h}+\phi$ is solution of

$$
\begin{gathered}
b\left(\xi_{h}+\phi,\left(v_{h}+\phi\right)-\left(\xi_{h}+\phi\right)\right) \geq\left(F^{\theta, k}+a_{0} \phi,\left(v_{h}+\phi\right)-\left(\xi_{h}+\phi\right)\right) \\
\xi+\phi \leq r_{h} \psi+\phi, \quad v_{h}+\phi \leq r_{h} \psi+\phi, \quad \forall v_{h} \in V^{h}
\end{gathered}
$$

Also, we have

$$
\begin{aligned}
F^{\theta, k} & \leq \tilde{F}^{\theta, k}+\left\|F^{\theta, k}-\tilde{F}^{\theta, k}\right\|_{\infty} \\
& \leq \tilde{F}^{\theta, k}+\frac{a_{0}}{\beta+\mu}\left\|F^{\theta, k}-\tilde{F}^{\theta, k}\right\|_{\infty} \\
& \leq \tilde{F}^{\theta, k}+a_{0} \phi,
\end{aligned}
$$

thus

$$
\partial_{h}\left(F^{\theta, k}, r_{h} \psi+\phi\right) \leq \partial_{h}\left(\widetilde{F}^{\theta, k}+a_{0}(x) \phi, r_{h} \tilde{\psi}+\phi\right) \leq \partial_{h}\left(\tilde{F}^{\theta, k}, r \tilde{\psi}\right)+\phi,
$$

hence

$$
\xi_{h} \leq \tilde{\xi}_{h}+\phi
$$

Similarly, interchanging the roles of $w$ and $\widetilde{w}$, we also get

$$
\tilde{\xi}_{h} \leq \xi_{h}+\phi .
$$

Finally, this yields 


$$
\begin{aligned}
\left\|\partial_{h}\left(F^{\theta, k}, r_{h} \psi\right)-\partial_{h}\left(\tilde{F}^{\theta, k}, r_{h} \tilde{\psi}\right)\right\|_{\infty} & \leq \frac{1}{\beta+\mu}\left\|F^{\theta, k}-\tilde{F}^{\theta, k}\right\|_{\infty} \\
& \leq \frac{1}{\beta+\mu}\left\|f^{\theta, k}+\mu w-f^{\theta, k}-\mu \tilde{w}\right\|_{\infty}\|w-\tilde{w}\|_{\infty} \\
& \leq \frac{1}{1+\beta \theta \Delta t}\|w-\tilde{w}\|_{\infty}
\end{aligned}
$$

which completes the proof.

Remark 4.3. If we set $0 \leq \theta<1 / 2$, the mapping $T_{h}$ is a contraction in $L^{\infty}(\Omega)$ with rate of contraction $2 /(2+\beta \theta(1-2 \theta) \rho(A))$, where $\rho(A)$ is a spectral radius of operator $A$.

Proof. Under condition of stability, we have shown the $\theta$-scheme is stable if and only if $\Delta t<$ $(2 C /(1-2 \theta)) h^{2}$.

Thus it can be easily show that

$$
\begin{aligned}
\left\|\partial_{h}\left(F^{\theta, k}, r_{h} \psi\right)-\partial_{h}\left(\widetilde{F}^{\theta, k}, r_{h} \tilde{\psi}\right)\right\|_{\infty} & \leq \frac{1}{1+\beta \theta \Delta t}\|w-\widetilde{w}\|_{\infty} \\
& \leq \frac{2}{2+\beta \theta(1-2 \theta) \rho(A)}\|w-\widetilde{w}\|_{\infty} \\
& \leq \frac{1}{1+\beta \theta\left((1-2 \theta) / 2 C h^{2}\right)}\|w-\tilde{w}\|_{\infty} \\
& =\frac{2 C h^{2}}{2 C h^{2}+\beta \theta(1-2 \theta)}\|w-\tilde{w}\|_{\infty}
\end{aligned}
$$

also it can be found that

$$
\begin{aligned}
\left\|\partial_{h}\left(F^{\theta, k}, r_{h} \psi\right)-\partial_{h}\left(\tilde{F}^{\theta, k}, r_{h} \tilde{\psi}\right)\right\|_{\infty} & \leq \frac{1}{1+\beta \theta\left((1-2 \theta) / 2 C h^{2}\right)}\|w-\tilde{w}\|_{\infty} \\
& =\frac{2 C h^{2}}{2 C h^{2}+\beta \theta(1-2 \theta)}\|w-\tilde{w}\|_{\infty}
\end{aligned}
$$

thus the mapping $T_{h}$ is a contraction in $L^{\infty}(\Omega)$ with rate of contraction $\left(2 C h^{2}\right) /\left(2 C h^{2}+\beta \theta(1-\right.$ $2 \theta)$ ). Therefore, $T_{h}$ admits a unique fixed point which coincides with the solution of P.V.I (4.7)

$$
\begin{aligned}
\left\|T_{h}(w)-\mathrm{T}_{h}(\tilde{w})\right\|_{\infty} & \leq \frac{1}{\beta+\mu}\left\|F^{\theta, k}-\tilde{F}^{\theta, k}\right\|_{\infty} \\
& =\frac{1}{\beta+\mu}\left\|f^{\theta, k}+\mu w-f^{\theta, k}-\mu \tilde{w}\right\|_{\infty} \\
& \leq \frac{\mu}{\beta+\mu}\|w-\tilde{w}\|_{\infty} \\
& \leq \frac{1}{1+\beta \theta \Delta t}\|w-\tilde{w}\|_{\infty} .
\end{aligned}
$$

This completes the proof. 


\subsection{Discrete Algorithm}

Starting from $u_{h}^{0}=u_{0 h}$ (initial data) and the $u_{h}^{\theta, 1}$ solution of problem (4.7), we introduce the following discrete algorithm:

$$
u_{h}^{\theta, k}=T_{h} u_{h}^{k-1}, \quad k=1, \ldots, n,
$$

where $u_{h}^{\theta, k}$ is the solution of the problem (4.7).

Remark 4.4. If we choose $\theta=1$ in (4.21), we get Bensoussan's algorithm. The idea of this choice has been studied by Boulbrachen (cf. [3]).

Proposition 4.5. Under the previous hypotheses, one has the following estimate of convergence: if $\theta \geq 1 / 2$

$$
\left\|u_{h}^{\theta, k}-u_{h}^{\infty}\right\|_{\infty} \leq\left(\frac{1}{1+\beta \theta \Delta t}\right)^{k}\left\|u_{h}^{\infty}-u_{h_{0}}\right\|_{\infty}
$$

and one has for

$$
\left\|u_{h}^{\theta, k}-u_{h}^{\infty}\right\|_{\infty} \leq\left(\frac{2 C h^{2}}{2 C h^{2}+\beta \theta(1-2 \theta)}\right)^{k}\left\|u_{h}^{\infty}-u_{h_{0}}\right\|_{\infty} \quad \text { for } 0 \leq \theta<\frac{1}{2} .
$$

Proof. we set a first case $\theta \geq 1 / 2$, and we have

$$
\begin{gathered}
u_{h}^{\infty}=T_{h} u_{h}^{\infty} \\
\left\|u_{h}^{\theta, 1}-u_{h}^{\infty}\right\|_{\infty}=\left\|T_{h} u_{h}^{0}-T_{h} u_{h}^{\infty}\right\|_{\infty} \leq\left(\frac{1}{1+\beta \theta \Delta t}\right)\left\|u_{h}^{0}-u_{h}^{\infty}\right\|_{\infty} .
\end{gathered}
$$

for $k \geq 2$, we use the Bensoussan-Lions' algorithm $\left(u_{h}^{k}=T_{h} u_{h}^{k-1}, k=1, \ldots, n\right)$ for a noncoercive elliptic quasivariational inequalities (cf., e.g., $[2,3])$ for details.

We assume that

$$
\left\|u_{h}^{\theta, k}-u_{h}^{\infty}\right\|_{\infty} \leq\left(\frac{1}{1+\beta \theta \Delta t}\right)^{k}\left\|u_{h}^{0}-u_{h}^{\infty}\right\|_{\infty}
$$

so

$$
\left\|u_{h}^{\theta, k+1}-u_{h}^{\infty}\right\|_{\infty}=\left\|T_{h} u_{h}^{k}-T_{h} u_{h}^{\infty}\right\|_{\infty} \leq\left(\frac{1}{1+\beta \theta \Delta t}\right)\left\|u_{h}^{k}-u_{h}^{\infty}\right\|_{\infty}
$$

thus

$$
\left\|u_{h}^{\theta, k+1}-u_{h}^{\infty}\right\|_{\infty} \leq\left(\frac{1}{1+\beta \theta \Delta t}\right)^{k+1}\left\|u_{h}^{0}-u_{h}^{\infty}\right\|_{\infty}
$$


for a second case $0 \leq \theta<1 / 2$, it can be easily shown that

$$
\left\|u_{h}^{\theta, k}-u_{h}^{\infty}\right\|_{\infty} \leq\left(\frac{2 C h^{2}}{2 C h^{2}+\beta \theta(1-2 \theta)}\right)^{k}\left\|u_{h}^{0}-u_{h}^{\infty}\right\|_{\infty} .
$$

\subsubsection{Asymptotic Behavior}

This section is devoted to the proof of main result of the present paper, where we prove the theorem of the asymptotic behavior in $L^{\infty}$-norm for parabolic variational inequalities

Now, we evaluate the variation in $L^{\infty}$ between $u_{h}^{\theta}(T, x)$, the discrete solution calculated at the moment $T=n \Delta t$ and $u^{\infty}$, the asymptotic continuous solution of (4.2)

Theorem 4.6 (The main result). Under condition of Theorem 4.1 and Proposition 4.5, one has for the first case $\theta \geq 1 / 2$,

$$
\left\|u_{h}^{\theta, n}-u^{\infty}\right\|_{\infty} \leq C\left[h^{2}|\log h|^{2}+\left(\frac{1}{1+\beta \theta \Delta t}\right)^{n}\right]
$$

and for the second case $0 \leq \theta<1 / 2$,

$$
\left\|u_{h}^{\theta, n}-u^{\infty}\right\|_{\infty} \leq C\left[h^{2}|\log h|^{2}+\left(\frac{2 C h^{2}}{2 C h^{2}+\beta \theta(1-2 \theta)}\right)^{n}\right]
$$

where $C$ is a constant independent of $h$ and $k$.

Proof. We have

$$
\left.u_{h}^{\theta, k}(x)=u_{h}(t, x) \quad \text { for } t \in\right](k-1) \Delta t ; k \Delta t[
$$

thus

$$
u_{h}^{\theta, n}(x)=u_{h}(T, x)
$$

then

$$
\begin{aligned}
\left\|u_{h}^{\theta}(T, x)-u^{\infty}\right\|_{L^{\infty}(\Omega)} & =\left\|u_{h}^{\theta, n}-u^{\infty}\right\|_{L^{\infty}(\Omega)} \\
& \leq\left\|u_{h}^{\theta, n}-u_{h}^{\infty}\right\|_{L^{\infty}(\Omega)}+\left\|u_{h}^{\infty}-u^{\infty}\right\|_{L^{\infty}(\Omega)} .
\end{aligned}
$$

Using, Theorem 4.1 and Proposition 4.5, we have for $\theta \geq 1 / 2$,

$$
\left\|u_{h}^{\theta, n}-u^{\infty}\right\|_{\infty} \leq C\left[h^{2}|\log h|^{2}+\left(\frac{1}{1+\beta \theta \Delta t}\right)^{n}\right]
$$


and for $0 \leq \theta<1 / 2$, we have

$$
\left\|u_{h}^{\theta, n}-u^{\infty}\right\|_{\infty} \leq C\left[h^{2}|\log h|^{2}+\left(\frac{2 C h^{2}}{2 C h^{2}+\beta \theta(1-2 \theta)}\right)^{n}\right] .
$$

\section{Conclusion}

In this paper, we have introduced a new approach for the theta time scheme combined with a finite element spatial approximation of parabolic variational inequalities (P.V.I). We have given a simple result to time energy behavior and established a convergence and asymptotic behavior in uniform norm. The type of estimation, which we have obtained here, is important for the calculus of quasistationary state for the simulation of petroleum or gaseous deposit. A future paper will be devoted to the computation of this method, where efficient numerical monotone algorithms will be treated.

\section{Acknowledgment}

The authors would like to thank the referee and the editors for reading and suggestions.

\section{References}

[1] A. Bensoussan and J.-L. Lions, Applications des Inéquations Variationnelles en Contrôle Stochastique, Dunod, Paris, Frnace, 1978.

[2] M. Boulbrachene, "Pointwise error estimates for a class of elliptic quasi-variational inequalities with nonlinear source terms," Applied Mathematics and Computation, vol. 161, no. 1, pp. 129-138, 2005.

[3] M. Boulbrachene, "Optimal $L^{\infty}$-error estimate for variational inequalities with nonlinear source terms," Applied Mathematics Letters, vol. 15, no. 8, pp. 1013-1017, 2002.

[4] S. Boulaaras and M. Haiour, " $L^{\infty}$-asymptotic bhavior for a finite element approximation in parabolic quasi-variational inequalities related to impulse control problem," Applied Mathematics and Computation, vol. 217, no. 14, pp. 6443-6450, 2011.

[5] P. Cortey-Dumont, "On finite element approximation in the $L^{\infty}$-norm parabolic obstacle variational and quasivariational inequalities," Rapport Interne 112, CMA. Ecole Polytechnique, Palaiseau, France.

[6] P. Cortey-Dumont, "On finite element approximation in the $L^{\infty}$-norm of variational inequalities," Numerische Mathematik, vol. 47, no. 1, pp. 45-57, 1985.

[7] P. Cortey-Dumont, "Approximation numérique d'une inéquation quasi variationnelle liée à des problèmes de gestion de stock," RAIRO_Analyse Numérique, vol. 14, no. 4, pp. 335-346, 1980.

[8] M. Haiour and S. Boulaaras, "Uniform convergence of schwarz method for elliptic quasi-variational inequalities related to impulse control problem," An-Najah University Journal for Research, vol. 24, pp. 71-89, 2010.

[9] J.-L. Lions and G. Stampacchia, "Variational inequalities," Communications on Pure and Applied Mathematics, vol. 20, pp. 493-519, 1967.

[10] P. G. Ciarlet and P.-A. Raviart, "Maximum principle and uniform convergence for the finite element method," Computer Methods in Applied Mechanics and Engineering, vol. 2, no. 1, pp. 17-31, 1973. 


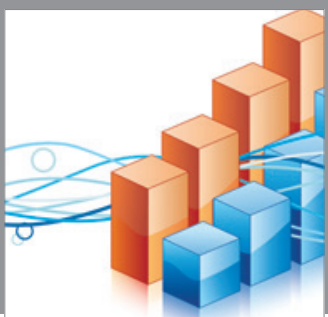

Advances in

Operations Research

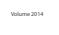

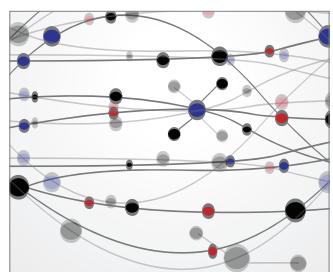

\section{The Scientific} World Journal
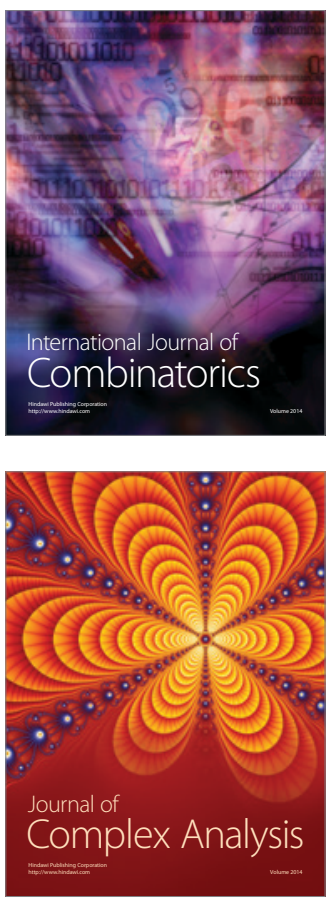

International Journal of

Mathematics and

Mathematical

Sciences
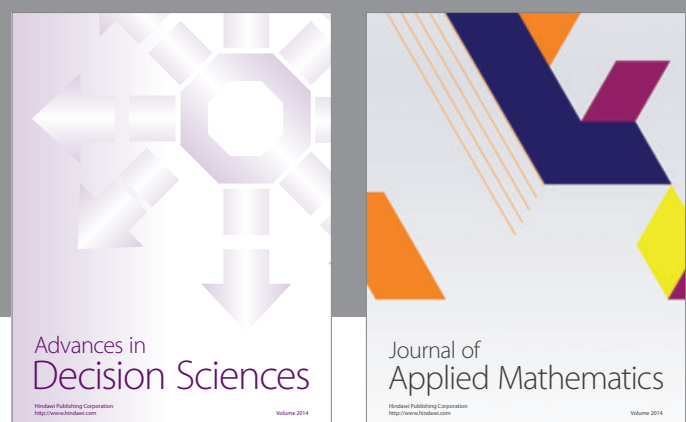

Journal of

Applied Mathematics
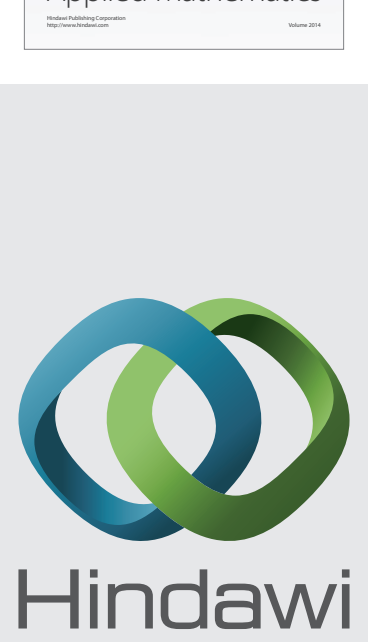

Submit your manuscripts at http://www.hindawi.com
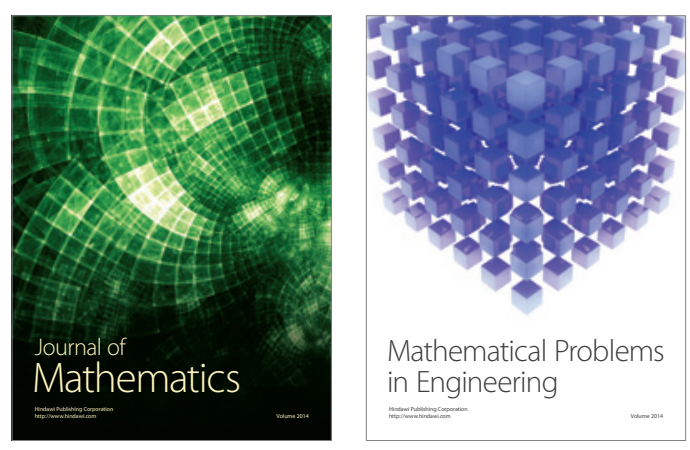

Mathematical Problems in Engineering
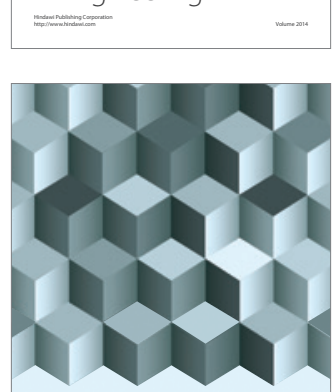

Journal of

Function Spaces
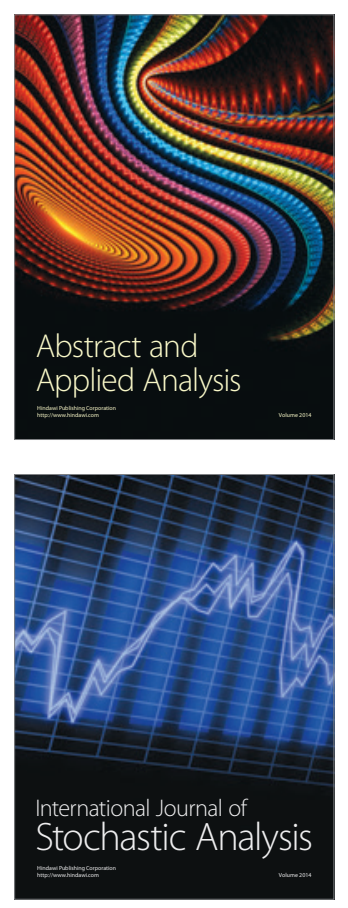

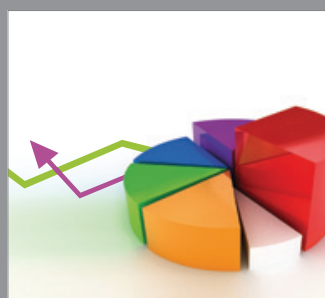

ournal of

Probability and Statistics

Promensencen
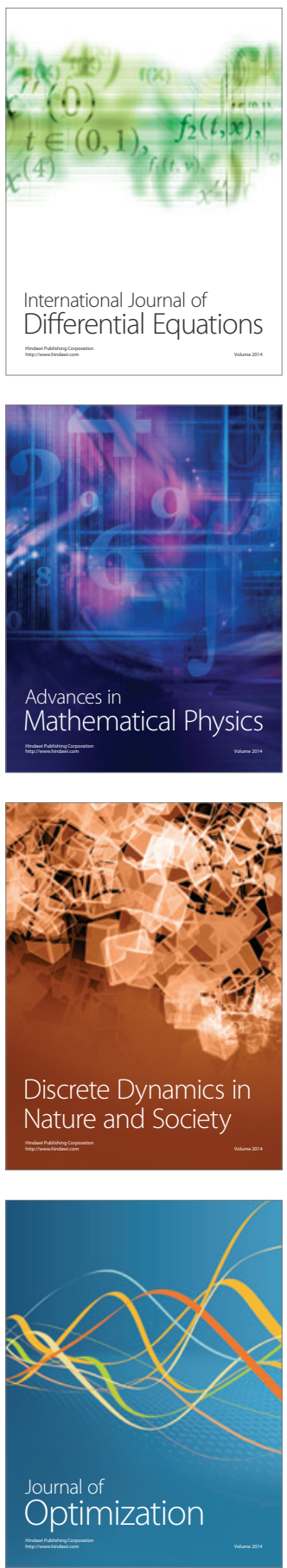